\title{
Selected Physical Therapy Program for Balance Training of Neuropathic Patients After Lower Limb Burn
}

\author{
Rasha Mohamed El-Rewainy ${ }^{1}$, Esraa Hany Rostom ${ }^{2}$, Hanan Helmy Elgendy ${ }^{3}$ \\ ${ }^{1}$ Department of Physical Therapy of Neuromuscular Disorders and Its Surgery, Faculty of Physical Therapy, Cairo University, Cairo, Egypt \\ ${ }^{2}$ Department of Physical Therapy for Surgery, Faculty of Physical Therapy, Cairo University, Cairo, Egypt \\ ${ }^{3}$ Department of Neurology, Faculty of Medicine, Cairo University, Cairo, Egypt
}

Email address:

Rasha85_elreweny@yahoo.com (R. M. El-Rewainy),Esraarostom@gmail.com (E. H. Rostom),

drhananelgendy76@gmail.com (H. H. Mohamed)

\section{To cite this article:}

Rasha Mohamed El-Rewainy, Esraa Hany Rostom, Hanan Helmy Elgendy. Selected Physical Therapy Program for Balance Training of Neuropathic Patients After Lower Limb Burn. Clinical Neurology and Neuroscience. Vol. 3, No. 2, 2019, pp. 35-42.

doi: $10.11648 /$ j.cnn.20190302.12

Received: March 19, 2019; Accepted: April 25, 2019; Published: June 18, 2019

\begin{abstract}
Background: Balance problems are very common with neuropathic patients following burn. Objective: To investigate the effect of selected physical therapy program on balance of neuropathic patients following burn. Methods: A randomized controlled study was conducted on fifty male patients (age range from 35 to 45 years) with polyneuropathy following burn injury of lower limb mainly at sole of the foot, $3^{\text {rd }}$ degree injury, axonotemesis with burn extent $30 \%$. Patients were selected from outpatient clinic, Faculty of physical therapy and Kasr AL Ainy hospital, Faculty of Medicine, Cairo University. The study was held during period between June 2017 to April 2018. The balance of patients was assessed before \& after treatment by Biodex balances system \& Berg Balance Scale. Patients were assigned randomly into two equal groups: Group A (study group) that received selected physical therapy program for balance training including (Stretching, Resistive and Balance exercises) and group B (control group) that received balance exercises only. Results: showed significant improvement in balance in favor to group A. Significant decrease in the balance indices and significant increase in BBS (Berg balance scale) scores were recorded after treatment in both study and control groups with better improvement of group A. Conclusion: On basis of the present data, it is possible to conclude that selected physical therapy program including (Stretching, Resistive and Balance exercises) is more effective for improving balance of neuropathic patients following burn than using balance exercises only.
\end{abstract}

Keywords: Burn, Polyneuropathy, Balance Training

\section{Introduction}

Poly-neuropathy is a common neuromuscular complication following burn. Patient suffers from pain; also there is affection of sensation and movement of the limbs. It was found that polyneuropathy is more common to occur with electrical and thermal burn. The frequency of polyneuropathy varies from $2 \%$ to $84 \%$ of patients [1].

Poly neuropathic patients often have balance disorders due to impairments that occur at sensory and motor abilities that results in frequent falls and postural instability especially with the closed eyes [2, 3].

Balance problems that occur with polyneuropathy caused by disturbances at proprioceptors. Other causes are movement-strategy impairment, biomechanical structural disorders, and disorientation [4].

Poly-neuropathy commonly occurs with thermal burns covering greater than $30 \%$ of the total body surface area and associated with axonotmesis than myelin sheath, and wallerian degeneration of axon have been found. The diagnosis of polyneuropathy is confirmed by significant reduction of motor and sensory nerve conduction velocity studies and decreasing mean caliber of large axons in histologic evaluation [5].

Flame injuries, and third degree injuries were the most common in patients with peripheral neuropathy. Axonotmesis was more common than demyelinating injury and polyneuropathy was more common than mononeuropathy [6].

An explanation to interpret how the thermal burn causes nerve injury is that thermal damage occurs as a result of burn 
causes denaturation and necrosis of the vessels and nerves. The vascular damage causes a partial-thickness burn that develops into a full-thickness burn due to diminishing of vascular supply to the burned area. In full thickness burns the nerves will be destroyed together with the overlying skin [7].

This study aimed to investigate the effect of selected physical therapy program on balance of neuropathic patients following burn.

\section{Methods}

The present study was held in the outpatient clinic, Faculty of Physical Therapy, Cairo University and Kasr AL Ainy hospital, Faculty of Medicine, Cairo University, during period between June 2017 to April 2018. This study was aiming to investigate the effect of selected physical therapy program on balance of neuropathic patients following burn. This is a randomized control trial, it was approved by the ethical committee of the faculty of physical therapy, Cairo University, Egypt (Approval Number: P.T.REC/012/002066).

\subsection{Subjects}

Fifty male patients with polyneuropathy following burn of lower limb mainly at sole of the foot, $3^{\text {rd }}$ degree injury, axonotemesis with burn extent $30 \%$ were randomly assigned into two groups, group A (the study group) $(n=25)$ and group $\mathrm{B}$ (the control group) $(\mathrm{n}=25)$ groups. Before testing, all subjects signed an informed consent form. All patients were subjected to a full clinical neurological assessment. Study participants were randomly allocated to either study group (GA) or control group (GB) by means of a random computergenerated list specific to each center. The randomization was managed by an external person uninvolved in the treatment. The method of allocation was concealed in sequentially numbered, sealed envelopes Figure 1.

\section{Consort Flow Diagram}

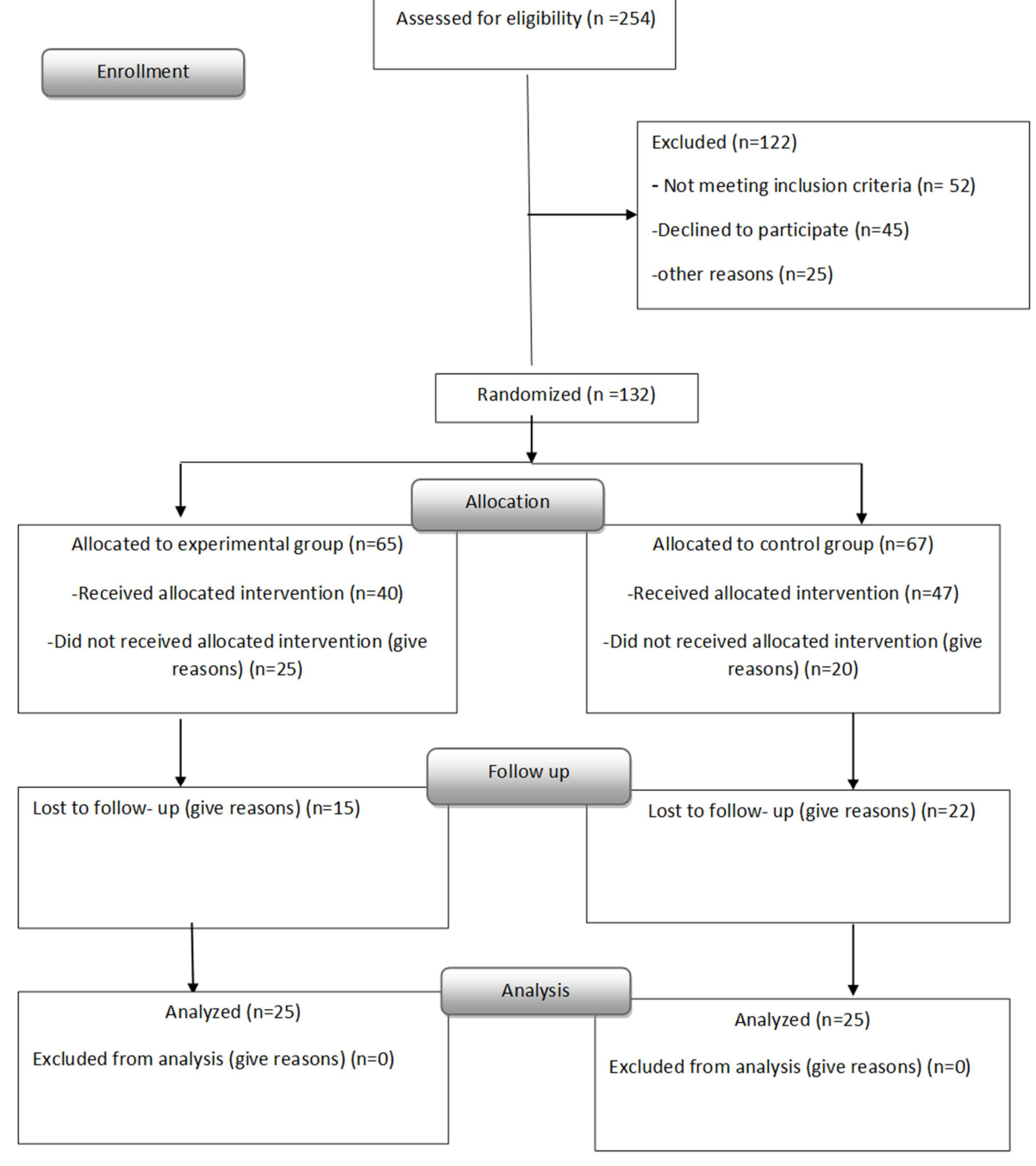

Figure 1. Study consort.

Patients in group A (study group) received selected physical therapy program for balance training including
(Stretching, Resistive and Balance exercises) and group B (control group) received balance exercises only. The 
treatment frequency was 3 times / week for 12 weeks (36 sessions).

Included in this study patients with polyneuropathy following burn injury of lower limb mainly at sole of the foot $3^{\text {rd }}$ degree injury, axonotmesis with burn extent $30 \%$. Patient's age ranged from 35 to 45 years. Patients were able to understand instructions and follow commands. Consent form was obtained from the patients.

Patients with the following were excluded: balance disturbance due to neurological disorders other than polyneuropathy (e.g. Parkinson's disease, inner ear, vestibular or cerebellar dysfunctions), patients with uncontrolled hypertension, patients with diabetes mellitus, patients with cognitive impairment or blindness, or deafness and patients with respiratory or cardiac patients.

\subsection{Assessment and Treatment Procedures}

A- Balance assessment procedures:

1-Biodex balance system

It is an important, effective and safe tool for balance and postural stability training that can be used for treating different neurologic diseases including neuropathic patients [8]' [9].

The Biodex balance system (Biodex Crop. Shirley, Ny, USA) consists of a circular platform that is free to move about the anterior-posterior (AP) and medial-lateral (ML) directions simultaneously. Also, the stability of the platform can be changed by varying the resistance force applied to the platform by underside springs. The springs' forces are adjustable to preset resistance levels that detect the difficulty levels of the Biodex balance system [10].

The indices which are assessed by the Biodex balance system include the following: Overall stability index (OSI), which is an index of deviation in any direction from the horizontal plane on the Biodex balance system plate; anterior-posterior stability index (APSI), which is an index of deviation on the Biodex balance system plate in the sagittal plane; and medial-lateral stability index (MLSI), which is an index of deviation on the Biodex balance system plate on the frontal plane [11].

A high score in the indexes indicates poor balance as this means that there is a great degree of deviation but as the patient becomes better, the score of the indices become lower [11].

Balance ability and all indices of both of study and control groups in different positions were measured with the Biodex Balance System (model 302-945; Shirley, New York).

Each subject was tested in six modes:

1-Standing on two legs with eyes both open and closed.

2- Standing on one leg at a time with eyes both open and closed.

3-Dynamic stability tests were conducted in each mode.

Testing of the patient with these tests and familiarization with the stabilometer machine should be done and this takes about 45 minutes for each patient.

2-Berg Balance Scale (BBS):

It is considered to be a clinical test of both static and dynamic balance abilities. It is a functional balance test and is considered to be the gold standard [12]. It is a sound measure of balance impairment and rehabilitation [13].

It consists of 14 simple balance related tasks, ranging from standing up from a sitting position, to standing on one foot. It takes from 15-20 minutes. Each task is given score from zero (unable or the lowest level of function) to four (Independent or the highest level of function), and the final measure is the sum of all of the scores of the tasks [14]. The higher score means that the patient has a better functional balance $[15,16]$.

Thus, the maximal overall score is 56 , and the minimal is zero. The higher score means that the patient has a better functional balance. The BBS has been shown to have excellent inter-rater and intra-rater relative reliability $[15,16]$

The risk of multiple falls is increasing below a score of 45 and a significant increase in multiple falls is below 40 [14]. The score of 45 is considered to be a proper cut-off for safe independent ambulation with the need for assistive devices or supervision. Patients with score above 45 to 56 are completely independent $[17,18]$.

B- Therapeutic procedures

Selected exercise program of ploy-neuropathic patient following burn:

1-Stretching Exercise: Stretching exercises are very important before doing other exercises especially for burn patients as those patients are very liable for developing tightness and contractures. Stretching exercises act to increase the flexibility of both of skin and muscles which are lost at burn patients [19].

Stretching exercises included calf muscle, hamstring muscle, Quadriceps muscle, Iliopsoas muscle.

Frequency of exercise: 3 times per week for 12 weeks with 30 minutes for each time [20].

Special considerations during exercising the burned patient:

*Pain - as the burned patient suffers from pain following burn injury, so during exercise the therapist has to use pressure garments on the burned areas in order to alleviate pain and to increase the patient ability to exercise.

*Dry skin - as the burned patient suffers from dryness of skin due to dehydration of water, so during exercising with the patient, the therapist has to put on creams to moisturize the skin of the patient especially before stretching to act to avoid cracking or tearing of skin. Also acting to make massage gently to the burned area helps a lot in smoothing the stretching.

*Water - as the burned patient suffers from dehydration, so the therapist has to inform him to drink large amounts of water and fluids to avoid dehydration during exercise.

*Exercising in the heat- burned patient are uncomfortable in the heat. So the therapist should inform the patient to avoid sun during exercising outdoors as he should wear hat and long sleeves, using waterproof sunscreen. The patient has to start the exercise slowly and should not spend long times in the heat. It was proved that burned patients with burns of less than $40 \%$ of total body surface area (TBSA) can build up a tolerance to the heat if they slowly increase exposure to the heat [19]. 
2-Resisted exercise: Resistive exercises act to increase power generated from lower limb muscles, also acts to increase the strength of lower limb muscles including hip flexors, knee flexors, knee extensors and ankle dorsiflxeors [21].

Resisted exercises that were given to hip, knee and ankle using weight such as cuff that was tied at the lower extremity according to the site of resistance, training was given by standardized oxford technique of progressive resistance exercises. 10 RM (ten repition maximum was determined by Brzyckis formula: $1 \mathrm{RM}=$ weight lifted during $\mathrm{n} \mathrm{RM} /$ (1.0278-.0278 (n)) and $10 \mathrm{RM}=75 \% 1 \mathrm{RM}[21]$.

Frequency of exercises: 3 times per week for 12 weeks. Rehabilitation Mode was Isotonic. Intensity was as follow: For 1st week: $50-60 \% 3 \mathrm{RM}$, for $2^{\text {nd }}$ to sixth week: $70-75 \%$ $3 \mathrm{RM}$ and for seventh to twelve weeks: $80-85 \% 3 \mathrm{RM}$. Volume was as follow: for $1^{\text {st }}$ to sixth week: $4-10$ reps and for seventh to twelve weeks: $8-12$ repetitions [22].

3-Balance exercise: Balance exercises improve subject's posture and balance by improving body awareness and control on subconscious level, so the quality of movement of the patient is improved [23].

First: Balance training exercises from sitting:

Second: Balance training from standing.

Third: Balance training from walking:

Frequency of exercises: 3 times per week for 12 weeks. Graduations of balance exercises from sitting to standing according to patient ability [24].

\subsection{Statistical Analysis}

Data were analyzed using the Statistical Program for Social Science (SPSS) version 22, IBM Corp., Chicago, USA, 2013. Quantitative data were expressed as mean \pm standard deviation (SD). Qualitative data were expressed as frequency and percentage. For quantitative data, independent $t$ test was used to compare between two independent variables with parametric data and paired t test to compare between two dependent variables. A one-way analysis of variance (ANOVA) was used to compare between more than two variables. Post hoc was used to test possible combinations of groups to determine where the significant differences are located. For qualitative data, Chi square was used for differences between proportions and Fisher exact for variables with small expected numbers. Pearson correlation coefficient was used for correlation between variables. A logistic regression analysis was performed to evaluate the independence of vitamin D role in the disease status. P-value $<0.05$ was considered significant and p-value $<0.01$ was considered highly significant.

\section{Result}

Demographic Data:

The age of the patients ranged from 35 to 45 years with a mean age of $39.2 \pm 3.278$ years for GA and $41 \pm 3.207$ for GB. The mean weight was $69.333 \pm 3.457$ for GA and $71.200 \pm 3.167$ for GB. The mean height was $160.60 \pm 1.844$ for GA and $159.67 \pm 1.952$ for GB. There was no significant difference between two groups in subjects' characteristics ( $\mathrm{P}>$ 0.05). Table 1

Table 1. Demographic data for age, weight, height.

\begin{tabular}{|c|c|c|c|c|}
\hline Variable & GA (Study group) $(n=25)($ Mean \pm SD $)$ & GB (Control group) $(n=25)($ Mean \pm SD $)$ & $\mathrm{t}$-value & P-value \\
\hline Age (yrs.) & $39.2 \pm 3.278$ & $41 \pm 3.207$ & 1.520 & $0.1397(\mathrm{NS})$ \\
\hline Weight (kg.) & $69.333 \pm 3.457$ & $71.200 \pm 3.167$ & 1.542 & $0.1343(\mathrm{NS})$ \\
\hline Height $(\mathrm{cm})$ & $160.60 \pm 1.844$ & $159.67 \pm 1.952$ & 1.346 & $0.1890(\mathrm{NS})$ \\
\hline
\end{tabular}

*Significant level is set at alpha level $<0.05 \mathrm{SD}$ : standard deviation, NS: Not significant

Concerning to Biodex indices:

Results showed that in Biodex indices, there was a statistical significant difference between the study(GA) and control group(GB) with better results for the study group concerning to overall stability index (Overall SI Angle / sec) the p- value post-test between GA and GB was 0.0001 . Table 2, Figure 2.

Table 2. Mean values of MLSI angle /sec-APSI angle / sec-Overall SI Angle / sec pre and post- test at both groups.

\begin{tabular}{|c|c|c|c|c|}
\hline Angle & & & & \\
\hline \multirow{2}{*}{ MLSI angle /sec } & Pre-test & Post test & \multirow{2}{*}{ t-value } & \multirow{2}{*}{ p- value } \\
\hline & Mean \pm SD & Mean \pm SD & & \\
\hline Group A & $5.087 \pm 0.2774$ & $4.033 \pm 0.2554$ & 10.819 & $0.0001 * * *$ \\
\hline Group B & $4.93 \pm 0.2717$ & $4.580 \pm 0.2569$ & 3.660 & $0.0010 * *$ \\
\hline t- value & 1.529 & 5.844 & & \\
\hline $\mathrm{p}$-value & $0.1374(\mathrm{NS})$ & $0.0001 * * *$ & & \\
\hline \multirow{2}{*}{ APSI angle / sec } & Pre-test & Post test & \multirow{2}{*}{$t$-value } & \multirow{2}{*}{ p-value } \\
\hline & Mean \pm SD & Mean \pm SD & & \\
\hline Group A & $6.205 \pm 0.1664$ & $5.193 \pm 0.1831$ & 15.841 & $0.0001 * * *$ \\
\hline Group B & $6.31 \pm 0.1791$ & $6.067 \pm 0.1915$ & 3.614 & $0.0012 * * *$ \\
\hline t- value & 1.679 & 12.767 & & \\
\hline $\mathrm{p}$-value & 0.1042 & $0.0001 * * *$ & & \\
\hline
\end{tabular}




\begin{tabular}{lllll}
\hline \multirow{2}{*}{ Overall SI Angle / sec } & Pre-test & Post test & t -value & p-value \\
\cline { 2 - 3 } & Mean \pm SD & Mean \pm SD & 26.707 & $0.0001^{* * *}$ \\
\hline Group A & $6.75 \pm 0.1246$ & $5.693 \pm 0.08837$ & 3.598 & $0.0012^{* * *}$ \\
Group B & $6.82 \pm 0.1859$ & $6.593 \pm 0.1580$ & & \\
t- value & 1.269 & 19.258 & & \\
$\mathrm{p}-$ value & 0.2149 & $0.0001^{* * *}$ & & \\
\hline
\end{tabular}

NS: Not significant, SD: Standard deviation, *Significant level is set at alpha level $<0.05$.

P-value: probability value **: Very significant.

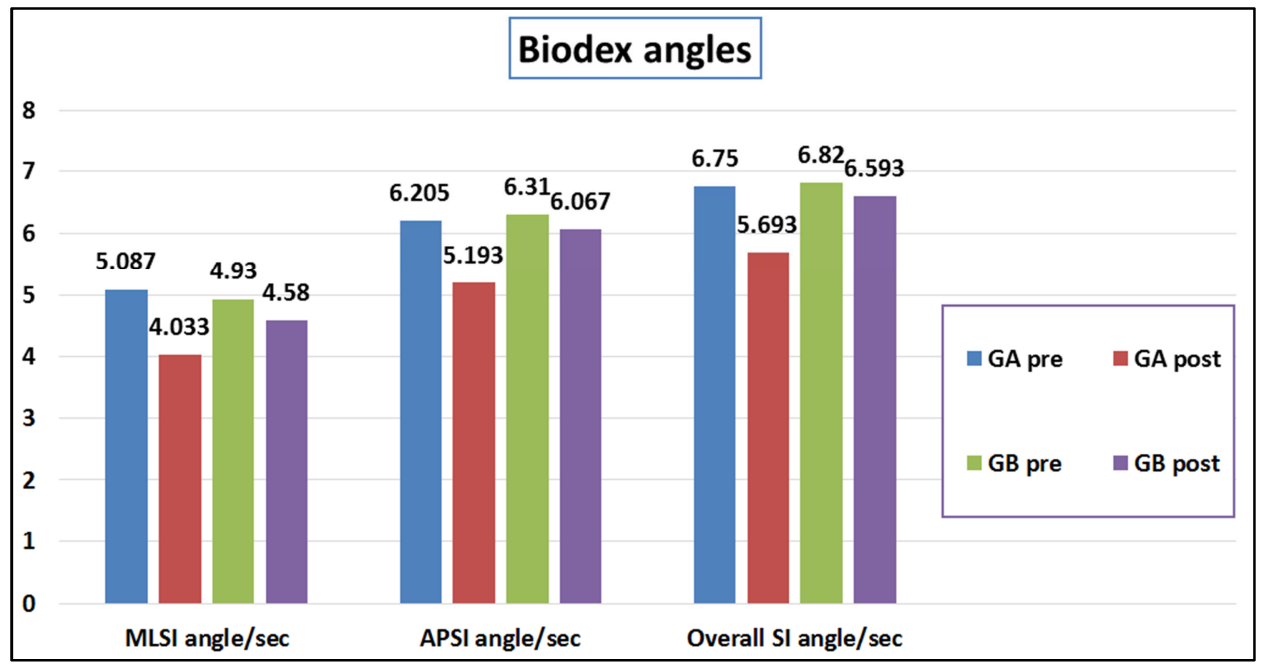

Figure 2. Mean values of MLSI angle /sec-APSI angle / sec-Overall SI Angle / sec pre and post- test at both groups.

Concerning to Berg balance scale:

Results showed that in Berg balance scale, there was a statistical significant difference between the study(GA) and control group $(\mathrm{GB})$ with favor to the study group $(\mathrm{GA})$ with the p-value post-test between GA and GB was 0.0001 . Table 3 , Figure 3 .

Table 3. Mean values of Berg Balance Scale pre and post test at both groups.

\begin{tabular}{lllll}
\hline \multirow{2}{*}{ BBS } & Pre test & Post test & t -value & p-value \\
\cline { 2 - 4 } & Mean \pm SD & Mean \pm SD & 15.002 & $0.0001^{* * *}$ \\
\hline Group A & $42.067 \pm 1.100$ & $48.267 \pm 1.163$ & 8.014 & $0.0001^{* * *}$ \\
Group B & $41.867 \pm 1.187$ & $44.800 \pm 0.7746$ & & \\
t- value & 0.4786 & 9.609 & & \\
p-value & 0.6359 & $0.0001^{* * *}$ & & \\
\hline
\end{tabular}

NS: Not significant, SD: Standard deviation, *Significant level is set at alpha level $<0.05$ P-value: probability value **: Very significant, BBS: Berg balance scale

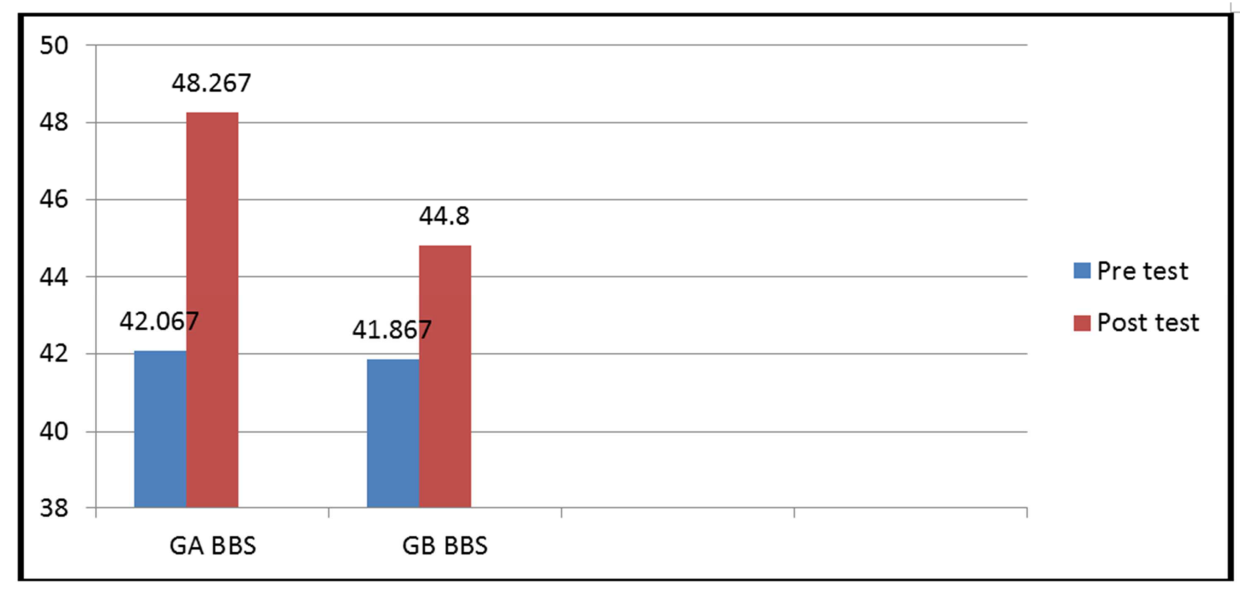

Figure 3. Mean values of Berg Balance Scale pre and post- test at both groups. 


\section{Discussion}

The present study aimed to investigate the effect of selected physical therapy program on balance of neuropathic patients following burn. The patients were collected from outpatient clinic in KASR-Alainy hospital and the study was held in the Faculty of Physical Therapy, Cairo University outpatient clinic; during period between June 2017 to April 2018.

Thirty male patients suffering from polyneuropathy following burn injury of lower limb with illness duration more than six months and age ranged between 35 and 45 years. The patients were assigned randomly into two equal groups; Group A (study group) that received selected physical therapy program for balance training including (Stretching, Resistive and Balance exercises) and group B (control group) that received balance exercises only.

Selected subjects were males as males are easier in dealing as they can transfer freely and attend sessions

The results of the present study showed that the selected physical therapy program of balance training including (stretching, resistive and balance exercises) was significantly more effective than training with balance exercises only in improving both of balance indices (OASI, APSI and MLSI), measured by Biodex balance system and Berg Balance Scale, in patients with poly-neuropathy following burn.

The results of the present study was supported by a study of Allet and his colleagues who stated that there was an improvement at balance, strength, walking speed and decreased recurrence of falling following training with a program including strength and balance training for 60 minutes, twice per week for a period of 12 weeks. It was proved that this program was very safe for persons with polyneuropathy [25].

Findings of this study also came in agreement with Fiona $\mathrm{P}$ who found that rehabilitation program including exercises (stretching, strengthening and balance), positioning and splinting for burned patients. Fiona stated that stretching of affected joints several times helps to maintain range of motion. Strength and balance exercises improve patient performance and functional capacity. Exercises also help the patient to experience a general feeling of wellbeing and a sense of confidence and achievement [26].

It was stated that combination of exercises including aerobic, stretching, strengthening, balance, proprioceptive and gait training was more effective for improving symptoms for neuropathic patients and improving nerve conduction velocity of nerves so improving function than training by only one kind of exercises [27].

A randomized controlled trial was conducted to study the effect of a physiotherapy intervention on polyneuropathy. This intervention was applied to focus on recovering range of motion, strength, and functionality of the joints most impaired by polyneuropathy. The main outcome is plantar pressure during foot rollover, and the secondary outcomes are kinetic and kinematic parameters of gait, neuropathy signs and symptoms, foot and ankle range of motion and function, muscle strength, and balance confidence. The intervention was carried out for 12 weeks, twice a week, for 40-60 min each session. The follow-up period is 24 weeks from the baseline condition. There was improvement in balance, muscle strength and range of motion [28].

It was found that combined resisted and balance exercises are more effective than resisted exercises only for improving proprioception and so improving balance, also these results are supported by the study results which stated that combined stretching, resisted and balance exercises are more effective for improving balance than balance exercises only for polyneuropathic patients following burn [29].

Another study proved that combination of exercises including balance and strength training was very beneficial for neuropathic patients [30].

The results of the present study come in contradict to the results of Fiona and his colleges who stated that balance training only is the most effective method for improving balance of polyneuropathic patients rather than combination of balance - strength - endurance exercises together [31].

Also, another study confirmed that there was an improvement at balance performance following balance training only in patients with neuropathy [23].

The results of the present study showed that patients trained by balance exercises only showed improvement in balance while patients trained by balance exercises, stretching and resistive exercises have shown better improvement in balance control

It can be concluded that balance training with selected physical therapy program including (balance, resistive and stretching exercises) was more effective for improving balance in neuropathic patients following burn than using balance exercises only. Limitations: Our study is limited because we have one type of patient (males only), so we recommend further studies including both sex (males and females).

\section{Conclusion}

On basis of the present data, it is possible to conclude that selected physical therapy program including (Stretching, Resistive and Balance exercises) is more effective for improving balance of neuropathic patients following burn than using balance exercises only.

\section{Acknowledgements}

The authors express their thanks to everyone who contributed to the article our dear colleagues in the outpatient clinic, all stroke patients, and relatives for their confidence and collaboration in this study and people who provided resources.

\section{Availability of Data and Materials}

The datasets generated and/or analyzed during the current 
study are not publicly available due to current Cairo University regulations and Egyptian legislation but are available from the corresponding author on a reasonable request and after institutional approval.

\section{Author Contributions}

RME wrote the paper, performed the statistical analysis, participated in the design, and collected the materials and data. EHR revised the paper and participated in the design and collection of data. HHM collected the materials and data and revised the paper.

\section{Competing Interests}

The authors declare that they have no competing interests.

\section{Funding}

The author(s) received no financial support for the research, authorship, and/or publication of this article.

\section{Authorship}

This work is original, has not been published and is not under consideration elsewhere.

\section{References}

[1] Fan kw, Zhuzx, Denzy. (2005). An experimental model of electrical injury to the peripheral nerve. Burns 31: 731-736.

[2] Ahmmed, A. U. and Mackenzie, I. J. (2003). Posture changes in diabetes mellitus. J Laryngol. Otol. 117(5): 358-364.

[3] Akbari, M., Karimi, H., Farahini, H. and Faghihzadeh, S. (2006). Balance problems after unilateral lateral ankle sprain. J Rehabil Res Dev. 43: 819-824.

[4] De Oliveira CB, De Medeiros IR, Frota NA, Greters ME, Conforto AB. (2008). Balance control in hemiparetic stroke patients; main tools for evaluation. J Rehabil Res Dev. 45(8):1215-26.

[5] Higashlmori H, Whetzel TP, Mahmood T, Carlsen RC. (2005). Peripheral axon caliber and conduction velocity are decreased after burn injury in mice. Muscle Nerve. 31: 610-620.

[6] Tamam Y, Tamam C, Tamam B and Ustundag M. (2013). Peripheral neuropathy after burn injury. Eur Rev Med Pharmacol Sci. 1:107-11.

[7] Coert JH. (2010). Pathophysiology of nerve regeneration and nerve reconstruction in burned patients. Burns. 36:593-8.

[8] Akbari, M., Jafari, H., Moshashaee, A. and Forugh, B. (2012). Do diabetic neuropathy patients benefit from balance training? J Rehabil Res Dev. 49: 333-338.

[9] Eftekharsadat, B., Babaei-Ghazani, A., Mohammadzadeh, M., Talebi, M., Eslamian, F. and Azari, E. (2015). Effect of virtual reality-based balance training in multiple sclerosis. Neurol Res. 37: 539-544.
[10] Schmitz, R. J. and Arnold, B. (1998). Interest and intratest reliability of a dynamic balance protocol using the Biodex stability system. J Sport Rehabil. 7 (2): 95-101.

[11] Arnold, B. and Schmitz, R. (1998). Examination of balance measures produced by the Biodex Stability System. J Athl Train. 33: 323-327.

[12] Langley, F. A. \& Mackintosh, S. F. H. (2007). Functional balance assessment of older community dwelling adults: A systematic review of the literature. The Internet Journal of Allied Health Sciences and Practice. 5(4).

[13] Blum, Lisa; Korner-Bitensky, Nicol. (2008). Usefulness of the Berg Balance Scale in Stroke Rehabilitation: A Systematic Review. Physical Therapy. 88 (5): 559-566.

[14] Muir SW, Berg K, Chesworth B, Speechley M.(2008). Use of the Berg Balance Scale for predicting multiple falls in community-dwelling elderly people: a prospective study. Phys Ther., 88 (4): 449-59.

[15] Downs S, Marquez J, Chiarelli P. (2013). The Berg Balance Scale has high intra- and inter-rater reliability but absolute reliability varies across the scale: a systematic review. $J$ Physiotherapy. 59: 93-99.

[16] Newstead, A. H., Hinman, M. R. and Tomberlin, J. A. (2005). Reliability of the Berg Balance Scale and Balance Master Limits of Stability Tests for Individuals with Brain Injury. Journal of Neurologic Physical Therapy. 29: 18-23.

[17] Boulgarides LK, McGinty SM, Willett JA, Barnes CW. (2003). Use of clinical and impairment-based tests to predict falls by community-dwelling older adults. Phys Ther. 83 (4): 328-39.

[18] Steffen T. M.; Hacker T. A., Mollinger L. (2002). Age- and Gender-Related Test Performance in Community-Dwelling Elderly People: Six-Minute Walk Test, Berg Balance Scale, Timed Up \& Go Test, and Gait Speeds." Journal of Physical Therapy. 82 (2): $128-137$.

[19] Meuleman, et. al. (2000). Exercise and training in the debilitated aged: Strength and functional outcomes. Arch phys Med Rehab. 81: 312-318.

[20] Cristina DS, Ricky W, Anice CP, Andreja PP, Renata HH and Isabel CS. (2012). Effects of a combined strengthening, stretching and functional training program versus usual-care on gait biomechanics and foot function for diabetic neuropathy: a randomized controlled trial. BMC Musculoskeletal Disorders J. 13: 36.

[21] Alvaro E Georg. (2005). Economic analysis of screening program for diabetes mellitus in Brazil. Rev Saude Publica. vol 39 (3):452-60.

[22] Hardee JP, Porter C, Sidossis LS, Borsheim E, Carson JA, Herndon DN. (2014). Early rehabilitative exercise training in the recovery from pediatric burn. Med Sci Sports Exerc.; 46:1710-6.

[23] Salsabili, H., Bahrpeyma, F., Forogh, B. and Rajabali, S. (2011). Dynamic stability training improves standing balance control in neuropathic patients with type 2 diabetes. J Rehabil Res Dev. 48: 775-786.

[24] Mueller MJ, Tuttle LJ, LeMaster JW, Strube MJ, McGill JB, Hastings MK. (2013). Weight-bearing versus non weightbearing exercise for persons with diabetes and peripheral neuropathy: a randomized controlled trial. Arch Phys Med Rehabil. 94: 829-838. 
[25] Allet L, Armand S, Golay A and Monnin D (2010). The gait and balance of patients with diabetes can be improved: a randomised controlled trial. Diabetologia. 53(3):458-466.

[26] Fiona P. (2010). Rehabilitation of the burn patient". Indian J Plast Surg. Sep. 43: 101-113.

[27] Shaker J and Steven MJ (2011). Update on the management of diabetic polyneuropathies. Diabetes Metab Syndr Obes. 4:289-305.

[28] Cristina D, Ricky W, Anice C and Andreja P (2012). Stretching and functional training program versus usual-care on gait biomechanics and foot function for diabetic neuropathy: a randomized controlled trial. BMC Musculoskeletal Disorders J. 13:36.
[29] Burak F and Bulkar B. (2016). The investigation of effect of balance and strength exercises on knee proprioception in young adolescents". British Journal of sports medicine.

[30] Cindy T, Constance V, and Donna L. (2017): " Strength and balance training for adults with peripheral neuropathy and high risk of fall: current evidence and implications for future research". PMC J. 39(5): E416-E424

[31] Fiona S, Eva M, Helmar C and Kathrin M. (2014). Exercise Intervention Studies in Patients with Peripheral Neuropathy: A Systematic Review". Sports medicine J. 44 (9): 1289-13. 